\title{
Effect of Varying Number of OSEM Subsets on PET Lesion Detectability
}

\author{
A. Michael Morey and Dan J. Kadrmas \\ Department of Radiology, Utah Center for Advanced Imaging Research (UCAIR), and Department of Bioengineering, \\ University of Utah, Salt Lake City, Utah
}

Iterative reconstruction has become the standard for routine clinical PET imaging. However, iterative reconstruction is computationally expensive, especially for time-of-flight (TOF) data. Block-iterative algorithms such as ordered-subsets expectation maximization (OSEM) are commonly used to accelerate the reconstruction. There is a tradeoff between the number of subsets and reconstructed image quality. The objective of this work was to evaluate the effect of varying the number of OSEM subsets on lesion detection for general oncologic PET imaging. Methods: Experimental phantom data were taken from the Utah PET Lesion Detection Database, modeling whole-body oncologic ${ }^{18} \mathrm{~F}-\mathrm{FDG}$ PET imaging of a 92-kg patient. The experiment consisted of 24 scans over $4 \mathrm{~d}$ on a TOF PET/CT scanner, with up to 23 lesions (diameter, 6-16 mm) distributed throughout the thorax, abdomen, and pelvis. Images were reconstructed with maximum-likelihood expectation maximization (MLEM) and with OSEM using 2-84 subsets. The reconstructions were repeated both with and without TOF. Localization receiver-operating-characteristic (LROC) analysis was applied using the channelized nonprewhitened observer. The observer was first used to optimize the number of iterations and smoothing filter for each case that maximized lesion-detection performance for these data; this was done to ensure that fair comparisons were made with each test case operating near its optimal performance. The probability of correct localization and the area under the LROC curve were then analyzed as functions of the number of subsets to characterize the effect of OSEM on lesion-detection performance. Results: Compared with the baseline MLEM algorithm, lesion-detection performance with OSEM declined as the number of subsets increased. The decline was moderate out to about 12-14 subsets and then became progressively steeper as the number of subsets increased. Comparing TOF with non-TOF results, the magnitude of the performance drop was larger for TOF reconstructions. Conclusion: PET lesion-detection performance is degraded when OSEM is used with a large number of subsets. This loss of image quality can be controlled using a moderate number of subsets (e.g., 12-14 or fewer), retaining a large degree of acceleration while maintaining high image quality. The use of more aggressive subsetting can result in image quality degradations that offset the benefits of using TOF or longer scan times.

\footnotetext{
Received Sep. 3, 2013; revision accepted Oct. 23, 2013.

For correspondence or reprints contact: Dan J. Kadrmas, 729 Arapeen

Dr., Salt Lake City, UT 84108-1218.

E-mail: kadrmas@ucair.med.utah.edu

Published online Nov. 12, 2013.

COPYRIGHT @ 2013 by the Society of Nuclear Medicine and Molecular Imaging, Inc.
}

Key Words: positron emission tomography (PET); OSEM; lesion detection; observer study; LROC

J Nucl Med Technol 2013; 41:268-273

DOI: 10.2967/jnmt.113.131904

$\mathbf{I}$ terative reconstruction algorithms that model Poisson statistics have become the standard for routine clinical PET imaging. Maximum-likelihood expectation maximization (MLEM) is the foundational algorithm; however, it is computationally expensive and requires many iterations to reach a suitable image. This problem is exacerbated by the emergence of time-of-flight (TOF) imaging, where the computational cost per iteration can be an order of magnitude slower than non-TOF (1). Block-iterative algorithms such as ordered-subsets expectation maximization (OSEM) are widely used to accelerate iterative image reconstruction (28). Here, the projection data are divided into subsets that are operated on sequentially during each OSEM iteration. The number of subsets provides the approximate acceleration factor-one iteration of OSEM with $N$ subsets provides an image roughly similar to that from $N$ iterations of MLEM (4-6). However, there is a tradeoff between the number of subsets and image quality. When the number of subsets is large, the size of each subset is small and each contains less tomographic and statistical information, potentially resulting in enhanced noise structures and other subset-related artifacts in the final image (4).

When OSEM is used in the clinic, it is important to understand the tradeoff between increasing the number of subsets (more acceleration) and image quality degradations (noise, artifacts). One approach would be to study how spatial resolution, contrast, and noise are affected by changing the number of subsets; however, these measures of image fidelity do not necessarily predict performance for clinical tasks. The accepted approach for objectively evaluating image quality in PET is to perform task-based assessments in which the different images are evaluated in terms of an observer's ability to perform a given task, such as detecting a lesion in the image. This task includes both detecting a lesion that is actually present (sensitivity) and correctly ruling out noise blobs that are not lesions (specificity) (9). The objective of 
this work was to evaluate the relationship between the number of OSEM subsets and image quality in terms of lesion detectability for general oncologic PET imaging with ${ }^{18} \mathrm{~F}-\mathrm{FDG}$.

Our group has established techniques for evaluating PET lesion-detection performance using specially designed phantom experiments (10-13), and these data and methodologies have been combined in a resource called the Utah PET Lesion Detection Database Resource (14). The resource consists of experimental data and routines for performing localization receiver-operating-characteristic (LROC) analysis (15-17) with the channelized nonprewhitened (CNPW) numeric observer (18). Model observers such as the CNPW have been shown to correlate with human observers for simple lesion-detection tasks $(10,11,18-24)$, and they offer the ability to quickly and repeatedly review large numbers of images. These data and LROC methods have previously been used to evaluate PET lesion-detection performance when modeling the point spread function (10), using TOF data (11), and reducing scan times (13).

In this work, experimental data from the Utah PET Lesion Detection Database were reconstructed with the MLEM algorithm (i.e., 1 subset) as a baseline and with OSEM using 11 different numbers of subsets (2-84 subsets). The reconstructions were repeated both with and without TOF data. Lesion-detection performance was assessed for each case using the CNPW observer with LROC analysis. The following sections describe the experimental data, reconstruction and data processing, LROC study methods, and results. The effect of increasing the number of OSEM subsets on lesion detectability is then analyzed, and conclusions based on the results are drawn.

\section{MATERIALS AND METHODS}

\section{Experimental Data for Lesion-Detection Assessment}

The lesion-detectability study used experimental data from the Utah PET Lesion Detection Database (14) for the custom large wholebody phantom scanned on a Biograph mCT TOF PET/CT scanner (Siemens Medical Solutions) with timing resolution of $527.5 \pm 4.9$ ps (25). The phantom, shown in Figure 1, had 3 main components: a 3-dimensional brain phantom; an anthropomorphic thorax phantom containing liver, lungs, and rib cage; and a pelvis with bladder compartment. The approximate dimensions of the phantom are $43 \times 28.0 \mathrm{~cm}$ at the widest points, and total length is approximately $83.1 \mathrm{~cm}$. Accounting for the missing mass of the arms and legs, the phantom models an approximately $92-\mathrm{kg}$ patient. The phantom also had several custom modifications designed to increase realism for modeling whole-body oncologic ${ }^{18}$ F-FDG PET (14).

The experiment consisted of 6 back-to-back whole-body scans acquired each day over the $4 \mathrm{~d}$ of the experiment. Each whole-body scan acquired list-mode data for 4 min per bed position over 6 bed positions. Three of the $4 \mathrm{~d}$ had $21-23$ shell-less ${ }^{68} \mathrm{Ge}$ (half-life, $270.8 \mathrm{~d}$ ) sources modeling lesions (26) with diameters of 6-16 mm distributed throughout the phantom lungs, liver, and soft-tissue compartments (mediastinum, abdomen, pelvis) to model tumors with focal ${ }^{18}$ F-FDG uptake. On the final day, no lesions were present, providing true-negative images for the observer study. This multiscan protocol provided numerous images and lesions with varying count levels and lesion target-to-background ratios. The

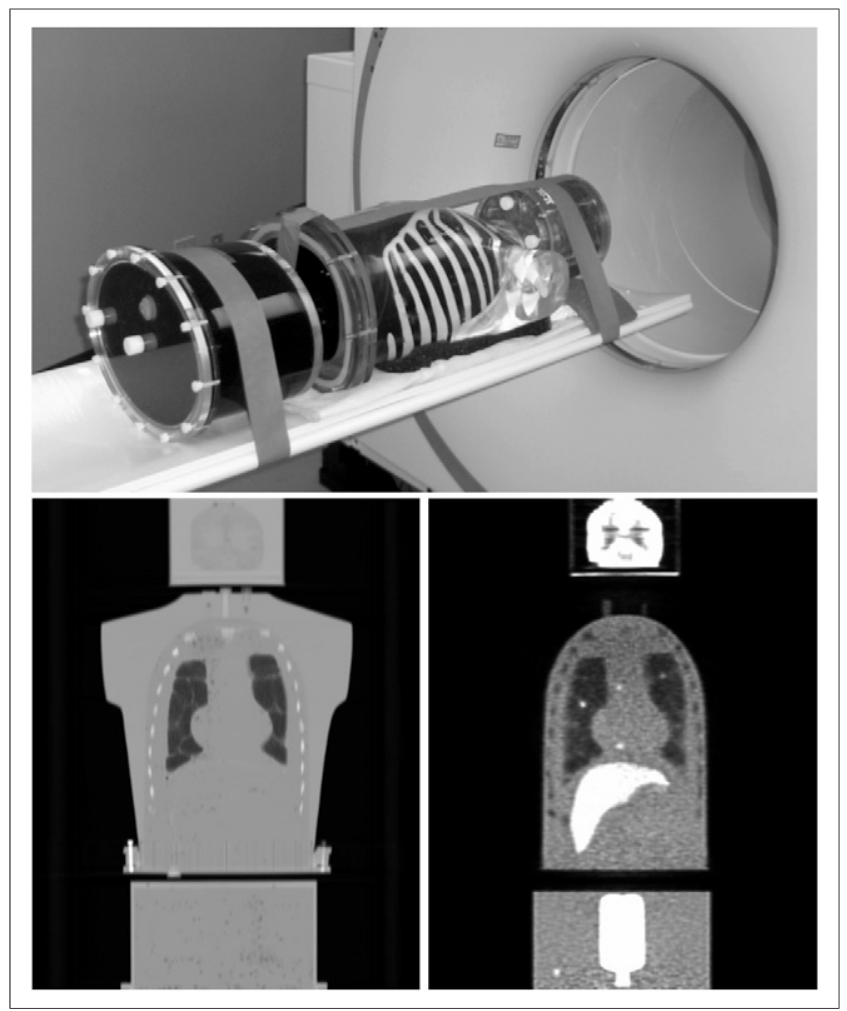

FIGURE 1. Whole-body phantom, shown on PET/CT scanner table (top), consists of brain compartment; thorax with liver, lungs, and rib cage/spine; and pelvis with bladder. It models patient of approximately $92 \mathrm{~kg}$. Coronal CT (bottom left) and PET (bottom right) images show main phantom compartments and structures. Example lesions can also be seen in PET image in both lungs, mediastinum, and pelvis regions.

overall activity levels for the 6 scans broadly covered the full range of activity levels representative of sites administering 370$555 \mathrm{MBq}$ of ${ }^{18} \mathrm{~F}$-FDG with uptake times ranging from 60 to $120 \mathrm{~min}$.

\section{Image Reconstruction and Processing}

The raw scan data, including list-mode files, attenuation maps, scanner calibrations, and scatter and randoms estimates, were loaded to an offline workstation and reconstructed using manufacturersupplied software (Siemens Medical Solutions). The baseline reconstruction algorithm was ordinary Poisson line-of-response MLEM with spatially variant point-spread function modeling (27), and each scan was reconstructed both with and without TOF. The reconstructed image matrix was $168 \times 168$, with $4.073-\mathrm{mm}$ pixels and 2.027-mm slice thickness. After reconstructing with MLEM, the reconstructions were repeated using OSEM with every available number of subsets. The sinogram data had 168 angles, and the reconstruction software required that the number of angles per subset be a multiple of 2 , giving the following numbers of subsets: 2, 3, 4, 6, 7, 12, 14, 21, 28, 42, and 84. Thus, 12 non-TOF and 12 TOF reconstructions were performed for each scan: MLEM and 11 versions of OSEM covering 2-84 subsets.

One challenge in comparing different OSEM reconstructions is that the rate of iterative convergence depends on the number of subsets, and similarly the noise properties (and hence the best postreconstruction filter) also depend on the number of subsets and iterations. To provide a fair comparison, it was important to objectively select the number of iterations and filter used for each case. The 
FIGURE 2. Example analysis results used for selecting number of iterations and filter strength for each case studied. Plot on left $(A)$ shows $A_{L R O C}$ vs. subiteration for MLEM and OSEM14 (where 1 subiteration represents 1 full pass through data; i.e., 1 iteration MLEM = 1 subiteration, and 1 iteration OSEM14 = 14 subiterations). Here, data are shown for filter that maximized $A_{L R O C}$ at each subiteration. Analogous plot on right (B) shows $A_{L R O C}$ vs. filter SD, where each datum is shown for number of iterations that maximized $A_{L R O C}$ for that filter strength. These data represent portion of multidimensional sampling used to optimize number of iterations and filter strength for phantom data used in this work.
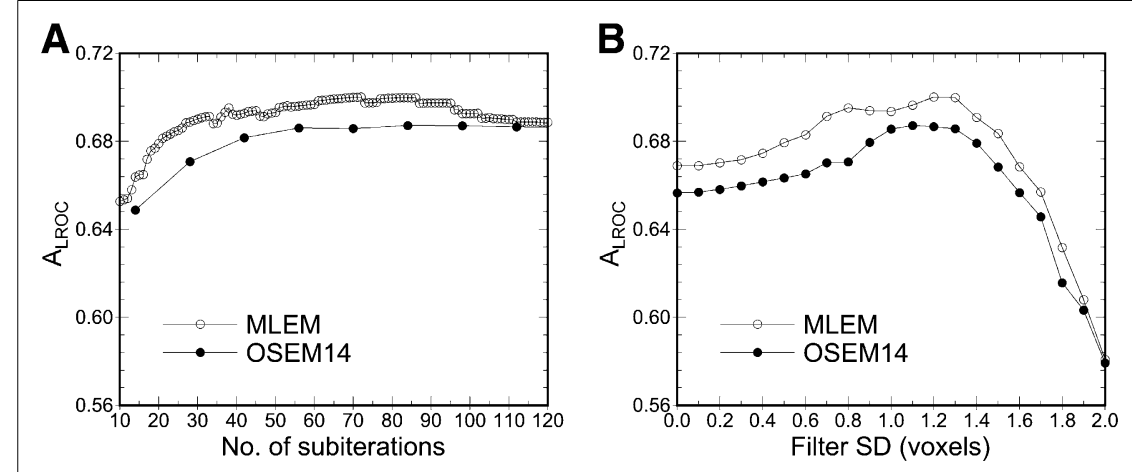

standard approach used with the Utah PET Lesion Detection Database (10-14) is to empirically optimize the number of iterations and postreconstruction filter for each algorithm that maximizes lesion-detection performance for that algorithm. As such, each algorithm was run out to at least 120 MLEM-equivalent iterations (e.g., 20 iterations for OSEM6), with a minimum of 10 iterations for each case, and the intermediate images from each iteration were stored for subsequent processing and analysis. The optimal number of iterations and smoothing filter were then selected using preliminary LROC studies.

The true location of each lesion in the phantom was determined from phantom setup coordinate grids and was confirmed on the CT scans. As reported previously (13), scans $2-5$ were found to provide the most clinically representative activity and noise levels, and data from these scans were used for the remainder of the study. This provided a total of 268 lesion-present test images (21-23 lesions $\times 4$ scans $/ \mathrm{d} \times 3 \mathrm{~d}$ with lesions present) plus 268 corresponding lesion-absent test images (from the scans without lesions) to be used for the LROC study for each reconstruction algorithm.

\section{LROC Analysis}

Preliminary LROC studies were first used to select the optimal number of iterations and postreconstruction filter for each algorithm, ensuring that each algorithm was fairly compared at nearmaximum performance. Here, 21 different 3-dimensional gaussian filters were applied to the images from each iteration, with SD ranging from 0.0 (no filter) to 2.0 voxels in 0.1 -voxel increments. The area under the LROC curve $\left(\mathrm{A}_{\mathrm{LROC}}\right)$ was computed for each iteration-filter combination. Figure 2 shows how $A_{\text {LROC }}$ changed as a function of iteration and filter for 2 TOF reconstruction cases and demonstrates that local changes to iteration and filter have minimal effects on $A_{\text {LROC. The iteration and filter that maximized }}$ $A_{\text {LROC }}$ were identified and selected for each algorithm; these values are listed in Table 1. These parameters maximized $A_{L R O C}$ for this particular set of experimental data, and they do not necessarily represent near-optimal or optimal parameters for clinical use. The topic of optimizing the number of iterations and filters for clinical use is large and complex and falls outside the scope of this work.

Empiric selection of the best number of iterations and filters required reading 7,834,176 test images to cover 268 lesion-present and lesion-absent test cases for each algorithm, iteration, and filter. It would not have been feasible to read this many images with human observers; however, the CNPW numeric observer completed this task within a few days. The CNPW observer computes a numeric rating, analogous to a human observer's confidence level, regarding the presence or absence of a lesion at each image location. The location with the highest rating was selected as the most probable lesion location for the LROC analysis. Additional details on the CNPW observer $(18,21)$ and its training and application to our experimental phantom data $(10-13)$ can be found in the references. As in this prior work, a radius of correct localization equal to 2.5 voxels was found to correctly identify hits while minimizing random localizations and was used throughout this study. Two figures of merit were used to quantify lesion-detection performance: the probability of correct localization $\left(\mathrm{P}_{\mathrm{LOC}}\right)$ and $\mathrm{A}_{\mathrm{LROC}} \cdot \mathrm{P}_{\mathrm{LOC}}$ is simply the fraction of lesions correctly localized within the 2.5 voxel threshold. A $A_{\text {LROC }}$ plots the correctly localized true-positive fraction versus the false-positive fraction, computed from the observer rating data and known truth. Higher values for these measures indicate higher lesion-detection performance.

\section{RESULTS}

\section{Example Images}

Example images reconstructed for each number of OSEM subsets are shown in Figure 3. The MLEM image provides the baseline for comparison and corresponds to OSEM with 1 subset. Increasing the number of subsets resulted in increased noise and subtle shape artifacts in these images,

TABLE 1

Selected Reconstruction Parameters

\begin{tabular}{rrrrr}
\hline & \multicolumn{2}{c}{ Non-TOF } & \multicolumn{2}{c}{ TOF } \\
\cline { 2 - 5 } $\begin{array}{l}\text { No. of } \\
\text { subsets }\end{array}$ & $\begin{array}{c}\text { No. of } \\
\text { iterations }\end{array}$ & $\begin{array}{c}\text { Filter SD } \\
\text { (voxels) }\end{array}$ & $\begin{array}{c}\text { No. of } \\
\text { iterations }\end{array}$ & $\begin{array}{c}\text { Filter SD } \\
\text { (voxels) }\end{array}$ \\
\hline 1 & 120 & 1.3 & 72 & 1.2 \\
2 & 58 & 1.3 & 36 & 1.2 \\
3 & 37 & 1.4 & 23 & 1.2 \\
4 & 24 & 1.2 & 21 & 1.2 \\
6 & 19 & 1.4 & 7 & 0.9 \\
7 & 18 & 1.4 & 13 & 1.3 \\
12 & 10 & 1.3 & 5 & 1.2 \\
14 & 10 & 1.5 & 6 & 1.1 \\
21 & 7 & 1.2 & 3 & 0.9 \\
28 & 7 & 1.4 & 3 & 1.2 \\
42 & 4 & 1.2 & 2 & 0.8 \\
84 & 5 & 1.3 & 1 & 0.8 \\
\hline
\end{tabular}




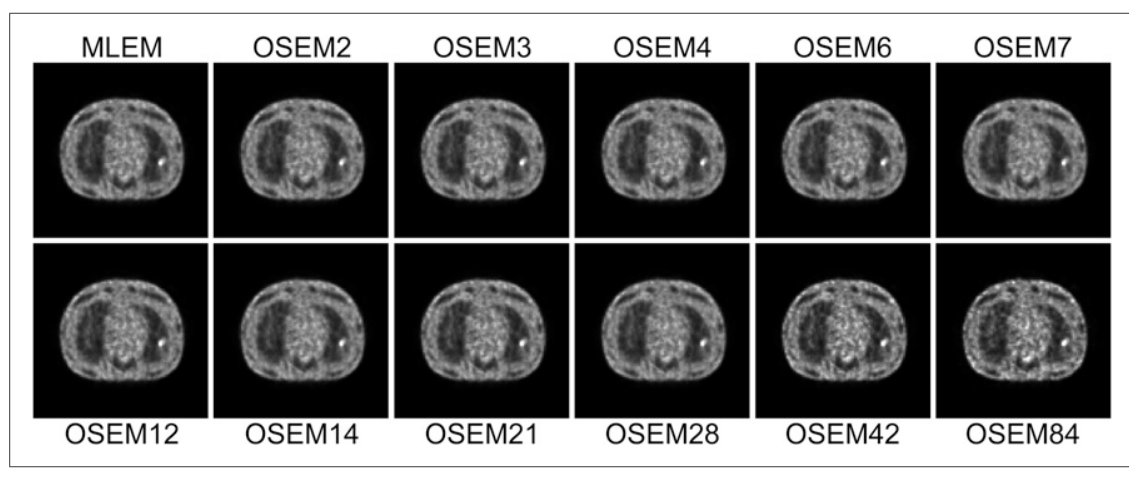

FIGURE 3. Example reconstructed images with TOF for each number of OSEM subsets, showing slice in mediastinum with 10-mm-diameter hot lesion in left lung. Each image is shown at approximately 56 MLEMequivalent iterations. Increasing noise and subtle shape-related artifacts can be observed in images as number of subsets increases. especially for the highest numbers of subsets. The overall objective of this work was to evaluate how these changes in the images affect lesion-detection performance for general oncologic PET imaging. Consider, for example, the sample images shown in Figure 4. This case had a true 8-mm lesion in the left lung and noise blobs of similar size and contrast in the mediastinum. The use of OSEM with 28 subsets resulted in lower contrast for the true (lung) lesion as compared with MLEM, coupled with increased contrast of the mediastinal noise blob. In this example, the CNPW observer correctly identified the lung lesion (true-positive) on the MLEM image but falsely identified the mediastinal noise blob (false-positive) on the OSEM28 image. This example illustrates how subset-related artifacts can affect lesion-detection performance.

\section{Lesion Detectability Versus Number of Subsets}

Figure 5 presents the main results of this paper, showing how $\mathrm{P}_{\mathrm{LOC}}$ and $\mathrm{A}_{\mathrm{LROC}}$ changed as functions of the number of OSEM subsets. Lesion-detection performance declined overall as the number of subsets increased. The decline was moderate out to about 12-14 subsets and then became progressively steeper as the number of subsets increased. When TOF and non-TOF results were compared, the same trend in performance was observed, but the magnitude of the performance drop was much larger for TOF. Overall, these results demonstrate that lesion-detection performance is only slightly degraded when a moderate number of subsets is used, suggesting that acceleration factors of as much as approximately 10 times can be safely attained with OSEM. However, more aggressive subsetting can cause more significant losses in image quality and adversely affect lesion detectability.

\section{DISCUSSION}

When LROC studies are performed, it is important to provide a context for interpreting the magnitude of differences in the figures of merit (i.e., in $\mathrm{P}_{\mathrm{LOC}}$ and $\mathrm{A}_{\mathrm{LROC}}$ ) in clinically relevant terms. The absolute magnitudes of $\mathrm{P}_{\mathrm{LOC}}$ and $\mathrm{A}_{\mathrm{LROC}}$ are determined largely by the experimental design. For example, one could include many large, highcontrast lesions that are easily detected-pushing the values of $\mathrm{P}_{\mathrm{LOC}}$ and $\mathrm{A}_{\mathrm{LROC}}$ close to one for all algorithms studied. Conversely, one could include many small, low-contrast lesions in the test dataset, resulting in $\mathrm{P}_{\mathrm{LOC}}$ and $\mathrm{A}_{\mathrm{LROC}}$ values closer to zero. Ideally, the test dataset would exactly model the clinically encountered distribution, in which case the absolute magnitude of the results would impart clinical meaning; however, such a distribution is not well understood and would vary widely by disease state. Furthermore, such a distribution would include many always-detectable lesions (found by all test algorithms) as well as many invisible lesions (e.g., micrometastases), neither of which would add to the statistical power of the study for differentiating the test algorithms. The lesion test data used here, as for most lesion-detectability

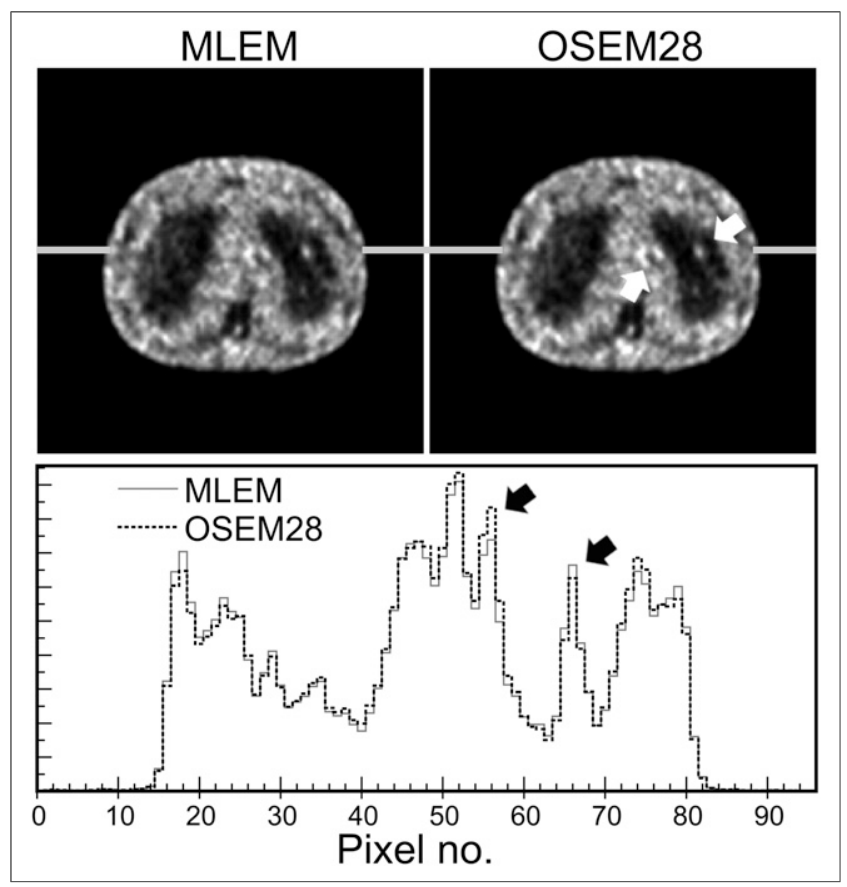

FIGURE 4. Example TOF images for MLEM and OSEM28 with optimal iteration and filter as determined by this study, demonstrating potential effects on lesion detectability. Focus in left lung is true 8-mm hot lesion, and foci in mediastinum are noise artifacts. Horizontal profiles showing relative intensity (arbitrary units) demonstrate that OSEM28 resulted in loss of contrast for lung lesion (right black arrow), coupled with increase in contrast for mediastinal noise blobs (left black arrow), as compared with MLEM. This example represents case in which observer identified correct (lung) lesion on MLEM image (i.e., true-positive reading) but misidentified mediastinal noise blob as lesion on OSEM28 image (i.e., false-positive). 
FIGURE 5. Lesion-detection performance, as measured by $P_{L O C}(A)$ and $A_{L R O C}(B)$, plotted as function of number of OSEM subsets for both TOF and non-TOF reconstructions. Performance declined overall as number of subsets increased, with marked drop in performance beyond approximately 28 subsets. For TOF reconstructions, performance drop at 42 subsets effectively canceled benefit of TOF.
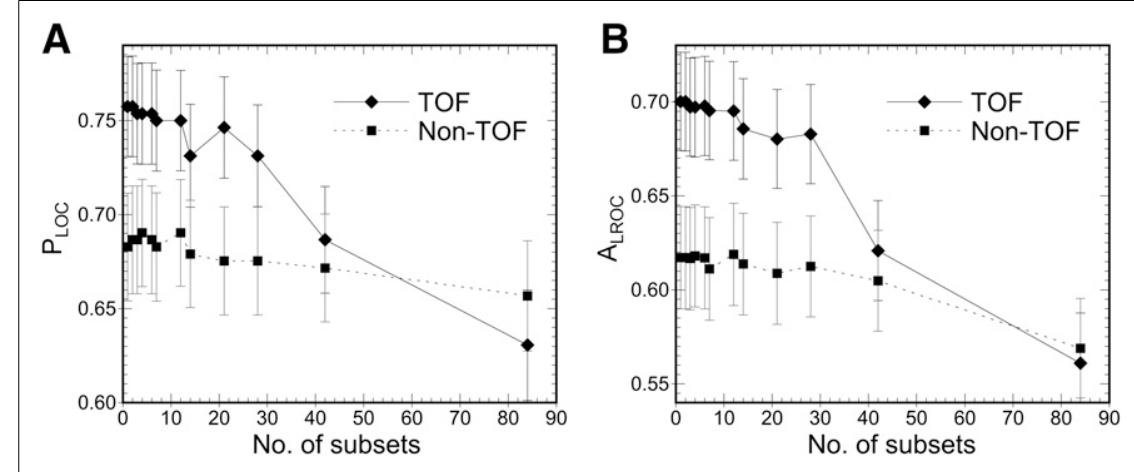

studies, were designed to provide high statistical power for differentiating and ranking the test algorithms studied. As such, the differences in the results should be interpreted within a meaningful context.

We provide 2 such contexts in this work. First, the impact of TOF versus non-TOF on PET lesion-detection performance has previously been evaluated in both phantoms and patients and is becoming well understood $(11,13,28-30)$. Comparing the TOF versus non-TOF results in Figure 5, one sees that the TOF reconstruction with 42 subsets provided approximately the same lesion-detection performance as the non-TOF reconstruction with MLEM. In essence, the degradation of using 42 subsets balanced and offset the benefit of using TOF in these data. The degradation from using 28 subsets cost approximately $20 \%$ of the benefit of TOF. Although these results should not be construed as exact quantifications, they do provide a context for assessing the significance of the changes observed in the results.

To provide additional context for interpreting the results, we repeated the MLEM TOF reconstructions and computed $A_{L R O C}$ as a function of scan time. Here, the raw list-mode PET data files were statistically pruned from $240 \mathrm{~s}$ per bed position to 180,120, and $90 \mathrm{~s}$ per bed position (corresponding to whole-body scan times of $24,18,12$, and $9 \mathrm{~min}$, respectively). The technique was the same as that presented in a previous publication (13). Repeating the LROC analysis for these images, we computed the change in $\mathrm{A}_{\mathrm{LROC}}$ as a function of scan time for MLEM. The results are shown in Figure 6, plotted alongside the results for changing the number of subsets. Here, using OSEM with 21 subsets was found to result in the same loss of detectability as found for MLEM when the scan time was reduced from 240 to approximately $205 \mathrm{~s}$ per bed position. Overall, these data suggest that reconstructing with OSEM up to about 12-14 subsets has only a moderate effect on lesion-detection performance but that using more subsets can result in more significant degradations.

\section{CONCLUSION}

When OSEM is used for tomographic reconstruction, the number of subsets provides the approximate acceleration factor for this algorithm as compared with MLEM. However, increasing the number of subsets also results in increased noise and subset-related artifacts in the image. This work evaluated the effect of changing the number of OSEM subsets on lesion-detection performance for general oncologic PET imaging. As compared with the baseline MLEM algorithm, lesion-detection performance declined as the number of OSEM subsets increased. The decline was moderate out to approximately 12-14 subsets for the data studied here, beyond which performance dropped more rapidly with the number of subsets. TOF PET reconstructions showed greater effect than non-TOF reconstructions. The degree of loss of lesion detectability with 21 subsets was similar to that observed when the scan time was reduced from 240 to $205 \mathrm{~s}$ per bed position. Similarly, the use of 42 subsets with TOF data offset the value of TOF, resulting in the same $A_{\text {LROC }}$ as non-TOF reconstructed with MLEM. We conclude that PET lesion-detection performance is degraded when OSEM

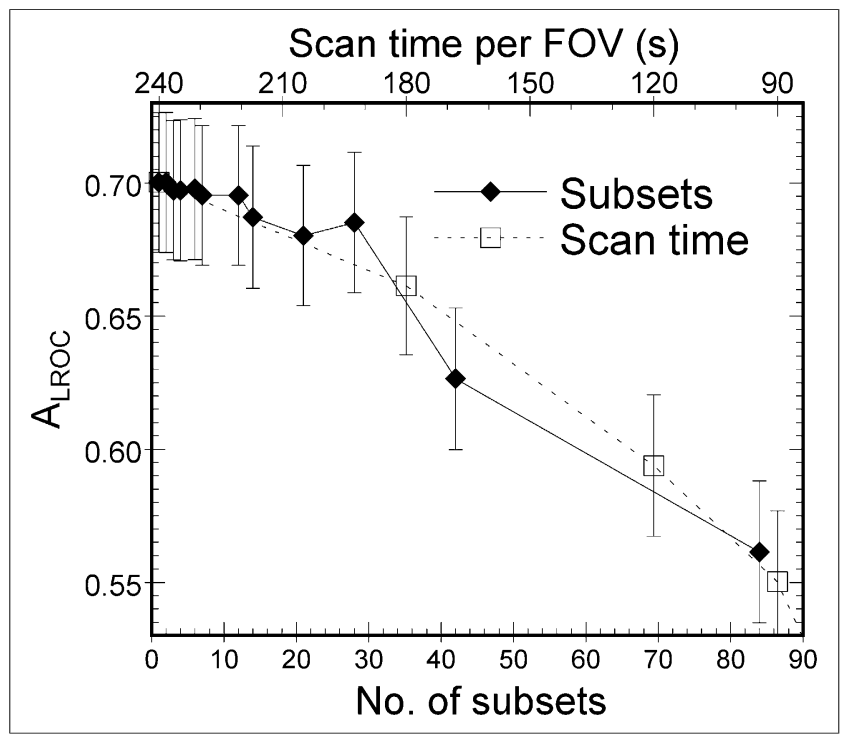

FIGURE 6. Comparison of how lesion-detection performance, as quantified by $A_{L R O C}$, is affected by increasing number of OSEM subsets or decreasing scan time for TOF reconstructions. These data provide context for interpreting significance of changes in $A_{L R O C}$ observed in this work. For example, use of approximately 32 subsets would result in same loss of performance as shortening scan time from 240 to $180 \mathrm{~s}$ per bed position. 
is used with a large number of subsets for both non-TOF and TOF reconstructions. This loss of image quality can be controlled by using a moderate number of subsets (e.g., 1214 or fewer), retaining a large degree of acceleration while maintaining high image quality.

\section{DISCLOSURE}

This work was supported by grant R03EB014454 from the National Institute of Biomedical Imaging and Bioengineering. Support for the experimental data was also provided in part by the National Cancer Institute under grant R01CA107353, by Siemens Medical Solutions, and by the Ben B. and Iris M. Margolis Foundation. No other potential conflict of interest relevant to this article was reported.

\section{ACKNOWLEDGMENT}

The authors would like to thank the anonymous reviewers, whose comments and suggestions greatly improved the paper.

\section{REFERENCES}

1. Pratx G, Surti S, Levin C. Fast list-mode reconstruction for time-of-flight PET using graphics hardware. IEEE Trans Nucl Sci. 2011;58:105-109.

2. Boellaard R, van Lingen A, Lammertsma AA. Experimental and clinical evaluation of iterative reconstruction (OSEM) in dynamic PET: quantitative characteristics and effects on kinetic modeling. J Nucl Med. 2001;42:808-817.

3. Dey J, King MA. Theoretical and numerical study of MLEM and OSEM reconstruction algorithms for motion correction in emission tomography. IEEE Trans Nucl Sci. 2009;56:2739-2749.

4. Hudson HM, Larkin RS. Accelerated image reconstruction using ordered subsets of projection data. IEEE Trans Med Imaging. 1994;13:601-609.

5. Hutton BF, Hudson HM, Beekman FJ. A clinical perspective of accelerated statistical reconstruction. Eur J Nucl Med. 1997;24:797-808.

6. Kadrmas DJ. Statistically regulated and adaptive EM reconstruction for emission computed tomography. IEEE Nucl Sci Symp Conf Rec. 2000;2:287-291.

7. Kadrmas DJ. LOR-OSEM: statistical PET reconstruction from raw line-of-response histograms. Phys Med Biol. 2004;49:4731-4744.

8. Liu X, Comtat C, Michel C, Kinahan P, Defrise M, Townsend D. Comparison of 3-D reconstruction with 3D-OSEM and with FORE+OSEM for PET. IEEE Trans Med Imaging. 2001;20:804-814.

9. Barrett HH, Myers KJ. Foundations of Image Science. Hoboken, NJ: John Wiley and Sons, Inc.; 2004

10. Kadrmas DJ, Casey ME, Black NF, Hamill JJ, Panin VY, Conti M. Experimental comparison of lesion detectability for four fully-3D PET reconstruction schemes. IEEE Trans Med Imaging. 2009;28:523-534.
11. Kadrmas DJ, Casey ME, Conti M, Jakoby BW, Lois C, Townsend DW. Impact of time-of-flight on PET tumor detection. J Nucl Med. 2009;50:1315-1323.

12. Kadrmas DJ, Christian PE. Comparative evaluation of lesion detectability for 6 PET imaging platforms using a highly reproducible whole-body phantom with ${ }^{22} \mathrm{Na}$ lesions and localization ROC analysis. J Nucl Med. 2002;43:1545-1554.

13. Kadrmas DJ, Oktay MB, Casey ME, Hamill JJ. Effect of scan time on oncologic lesion detection in whole-body PET. IEEE Trans Nucl Sci. 2012;59:1940-1947.

14. Kadrmas DJ. The Utah PET lesion detection database. IEEE Nucl Sci Symp Conf Rec. 2009:3693-3699.

15. Khurd P, Gindi G. Fast LROC analysis of Bayesian reconstructed emission tomographic images using model observers. Phys Med Biol. 2005;50:1519-1532.

16. Popescu LM. Nonparametric ROC and LROC analysis. Med Phys. 2007;34: 1556-1564.

17. Khurd P, Gindi G. Rapid computation of LROC figures of merit using numerical observers (for SPECT/PET reconstruction). IEEE Trans Nucl Sci. 2003;4:25162520 .

18. Gifford HC, Kinahan PE, Lartizien C, King MA. Evaluation of multiclass model observers in PET LROC studies. IEEE Trans Nucl Sci. 2007;54:116-123.

19. Myers KJ, Barrett HH. Addition of a channel mechanism to the ideal-observer model. J Opt Soc Am A. 1987;4:2447-2457.

20. Gifford HC, King MA, de Vries DJ, Soares EJ. Channelized hotelling and human observer correlation for lesion detection in hepatic SPECT imaging. J Nucl Med. 2000;41:514-521.

21. Gifford HC, King MA, Pretorius PH, Wells RG. A comparison of human and model observers in multislice LROC studies. IEEE Trans Med Imaging. 2005; 24:160-169.

22. Fiete RD, Barrett HH, Smith WE, Myers KJ. Hotelling trace criterion and its correlation with human-observer performance. J Opt Soc Am A. 1987;4:945-953.

23. Barrett HH, Yao J, Rolland JP, Myers KJ. Model observers for assessment of image quality. Proc Natl Acad Sci USA. 1993;90:9758-9765.

24. Lartizien C, Kinahan PE, Comtat C. Volumetric model and human observer comparisons of tumor detection for whole-body positron emission tomography. Acad Radiol. 2004;11:637-648.

25. Jakoby BW, Bercier Y, Conti M, Casey ME, Bendriem B, Townsend DW. Physical and clinical performance of the mCT time-of-flight PET/CT scanner. Phys Med Biol. 2011;56:2375-2389.

26. Hamill JJ, Arnsdorff CE, Casey ME, Xinli L, Raulston WJA. Ge-68 PET hotsphere phantom with no cold shells. IEEE Nucl Sci Symp Conf Rec. 2005;3: 1609-1613.

27. Panin VY, Kehren F, Michel C, Casey M. Fully 3-D PET reconstruction with system matrix derived from point source measurements. IEEE Trans Med Imaging. 2006;25:907-921.

28. Karp JS, Surti S, Daube-Witherspoon ME, Muehllehner G. Benefit of time-offlight in PET: experimental and clinical results. J Nucl Med. 2008;49: $462-470$.

29. Surti S, Scheuermann J, El Fakhri G, et al. Impact of time-of-flight PET on whole-body oncologic studies: a human observer lesion detection and localization study. J Nucl Med. 2011;52:712-719.

30. El Fakhri G, Surti S, Trott CM, Scheuermann J, Karp JS. Improvement in lesion detection with whole-body oncologic time-of-flight PET. J Nucl Med. 2011;52: 347-353. 